Ophthalmologica 1967;154:I-VI

\title{
Contents, Vol. 154, 1967
}

\section{Index}

Alfonso, G. F.: vide Molfetta, V. be

Amalric, P. (Albi/Tarn):

Intérêt du test à la fluorescéine dans $\Gamma$ étude de certaines dégéné-rescences tapéto-rétiniennes et en particulier dans le fundus flavi-maculatus 367

Aouchiche, M.; Laliam, M. et Ismail-Dahlouk, M. (Alger):

Naevus fusco coeruleus ophtalmo-maxillaire de Ota 217

Babel, J.: $\quad$ vide Vicari, S.

Barraquer, J. L; Barraquer, J. und Littmann, H. (Barcelona):

Ein besonders geeignetes Operationsmikroskop für die Augen-chirurgie 483

Barraquer, J.: vide Barraquer, J. I.

Bert, J.: $\quad$ vide Graveline, J.

Binkhorst, C. D. and Gobin, M. H. (Terneuzen):

Pseudophakia after Lens Injury in Children 81

Blechschmidt, E. (Göttingen):

Die Entwicklungsbewegungen der menschlichen Retina zur Zeit derIrisentstehung. Die

Entstehung des Ganglion opticum als Beispieleiner submikroskopisch untersuchbaren

Entstehung einer Cyto-architektonik 531

Breitenmoser, R. (Zurich):

Auto-Ophthalmoskopie am Orthoskop 521

Brienen, J. A. and Snell, C. A. R. D. (Utrecht):

A New Examination of the Orbicularis Oculi 104

Cardinet, J.: vide Vicari, S.

Castro-Correia, J. (Porto):

Studies on the Innervation of the Uveal Tract

Coster, H. D.: vide Dekking, H. M.

Daicker, B. (Basel):

Iuxtavenöse degenerative Grubenbildungen der Netzhaut .... 286

Dekking, H. M. and Coster, H. D. (Groningen):

Dynamic Tonometry 59

Dos Santos, J.: vide Moutinho, $\mathrm{H}$.

Dubroff, S.: vide Green, W. R.

Dufour, R. (Lausanne):

Le point de vue medical dans le traitement et $\Gamma$ éducation desaveugles

Dufour, R.: vide Pannatier, J.

Eggers, C. (Santiago de Chile):

Dystrophia cornealis posterior polymorpha (Hornhautdystrophie

von Schilling) 1

Eisner, G. (Bern): Traumatische Veränderungen der Pars plana corporis ciliaris. . . . 312 
Esslen, E. und D. und Huber, A. (Zurich):

Elektromyographische Innervationsanalyse des Strabismus con-comitans 189

Faggioni, R.: vide Stucchi, C. A.

Fanta, H. (Wien): Ansprache im Namen der Österreichischen Augenärztegesellschaft . 188

Fekker, Olga (Miami, Fla.):

Two Years Fluorescein Follow-up of Diabetic Microaneurysms. . . 6

Fkanceschetti, A. (Geneve):

Presentation de malades de la Clinique d'ophtalmologie de Geneve . 431 Francois, J.; Vekbeke,

J. et Verluyten, P. (Gand):

Guérison d'une pseudothrombose dysprotéinémique par la théra-

peutique cytostatique et corticoïde 392

Francois, J.; Goes, F. et Yobbágyi, P. (Gand):

Etude biométrique d'une ectasie sclérale péripapillaire congénitale

(staphylome postérieur vrai) 446

Francois, J. et Victoria-Troncoso, V. (Gand):

La conjonctivite ligneuse $\quad 470$

Gerhard, J. P. (Strasbourg):

Etude du cuivre de Thumeur aqueuse chez une famille atteinte de

maladie de Wilson 405

Gernet, H. (Münster):

Über relative und absolute Häufigkeit der Netzhautablösung bei

Myopie $\quad 373$

Gnädinger, M. (Basel):

Die Acetylcholin-Spaltungsfähigkeit des Epithels der Kaninchen-

hornhaut 295

Gobin, M. H.: vide Binkhorst, C. D.

Goes, F.: vide Francois, J.

Goldmann, H. (Bern):

Glaskörperabhebung 324

Goswami, A. P. (Kanpur):

Ecbolics and Congenital Eye Defects 441

Graveline, J.; Quéré, M. A. (Bourg la Reine, Seine) et Bert, J. (Dakar):

L'électrorétinogramme chez un lémurien nocturne: le Galago

senegalensís 143

Green, W. R.; Kaufer, G. J. and Dubroff, S. (Washington, D.C.):

Black Cornea. A Complication of Topical Use of Epinephrine ... 88

Haase, E.: vide Müller, W.

Hilsdorf, C. (St. Gallen):

Über das Vorkommen von Drusen bei Atrophia gyrata chorioideae

et retinae $\quad 361$

Huber, A.: $\quad$ vide Esslen, E.

Huber, O. (Glarus):

Wandlungen in der Ophthalmologie 1916-1966 401

Ismail-Dahlouk, M.:

vide Aouchiche, M.Iwamoto, T.: $\quad$ vide Witmer, R.

Jaeger, W. (Heidelberg): 
Ansprache anläßlich der Ehrenpromotion von Professor Dr. med.

A. Franceschetti zum Dr. med. h.c. der Universität Heidelberg. . 185 Junod, Anne-Marie

(Olten):

Soziale Fragen der Blindenbetreuung 259

Kahán, A. und Kahán, I. L. (Szeged):

Über die Bedeutung der Erythrozyten-Lipoide in manchen vasku-

lären Vorgängen des Augenhintergrundes 573

Kahán, I. L. and Vass, Z. (Szeged):

Nature of Human Lens Lipids. Analytical (Quantitative and Thin-Layer Chromatographic) and Histochemical Investigations .... 551

Kahán, I. L.: vide Kahán, A.

Kaufeh, G. J.: vide Green, W. R.

Kern, R. (Zurich): Experimentelles zum Cortisonglaukom. 2. Eine in lutro-Studie

anintraokulären Muskelstreifen des Rhesusaffen mit steroidhaltigenPräparaten

Laliam, M.: vide Aouchiche, M.

Landolt, E. (Zurich):

Atypisches phakolytisches Glaukom 334

Lang, J. (Zurich): Der kongenitale oder frühkindliche Strabismus 201

Lax, F.: $\quad$ vide Reim, M.

Lichte, H.: $\quad$ vide Reim, M.

Littmann, H.: vide Barraquer, J. I.

Maione, M.; Maraini, G. and Pasino, L. (Parma):

Variability of Responses to Different Methods of Examination inAnomalous Retinal

Correspondence $\quad 96$

Maraini, G.: vide Maione, M.

Martenet, A. C.; Witmer, R. et Speiser, P. (Zurich):

Alterations oculaires dans Thomocystinurie 318

Martenet, A. C.: vide Witmer, R.

Maselli, E.: vide Spineixi, D.

Meulemans, G. (Bruxelles):

Les fractures de la region orbito-maxillo-malaire $\quad 461$

Meyer, R. (Lausanne):

Reconstructions orbito-palpébrales par lambeau frontotemporal . . 328

Miglior, M.; Redi, F.; Spinelli, D.; Molfetta, V. de et Polenghi, F. (Milan):

La rétinopathie pigmentaire en secteurs symétriques. Apportclinique

Molfetta, V. de; Spinelli, D. et Alfonso, G. F. (Milan):

Recherches sur des potentiels électriques oculaires obtenus par stimulation lumineuse de Toeil controlatéral 354

Molfetta, V. de : vide Miglior, M.

Molfetta, V. de : vide Spinelli, D.

Molnár, L. (Debrecen):

Zur Verwendbarkeit der Bifokalbrille bei akkommodativen Schielenden 128

Moutinho, H. et Dos Santos, J. (Lisbonne):

Quelques aspects médico-sociaux de la rehabilitation des aveugles . 252

Müller, W. und Haase E. (Erfurt):

Der Einfluß der Präadaptation auf das bei Tageslicht abgeleiteteElektroretinogramm 
Niesel, P.: $\quad$ vide Patnaik, B.

Orbán. T. (Budapest):

Histologischer Befund einer gegen Ablatio retinae ausgeführtenSkleraresektion 135

Otto, J.(St.Gallen): Binokularschulung des Strabismus alternans ohne ausgebildete ARC 114

Otto, J.(St.Gallen): Schulungsmöglichkeiten bei organisch bedingter hochgradiger Sehminderung 209

Pannatier, J. et Dufour, R. (Lausanne):

Experience au Foyer, centre éducatif pour aveugles intellectuellement

handicapés 270

Pasino, L.: vide Maione, $\mathrm{M}$.

Patnaik, B. und Niesel, P. (Bern):

Tyndallometrie in der Vorderkammer mit Hilfe der Spaltlampen-

photographie 423

Polenghi, F.: vide Miglior, M.

Polychronakos, D. and Razoglou, Ch. (Thessaloniki):

Treatment of Total Hyphema with Fibrinolysin

Quéré, M. A.: vide Graveline, J.

Rabinovicz, Th. : vide Stucchi, C. A.

Razoglou, Ch. : vide Polychronakos, D.

Redi, F.: $\quad$ vide Miglior, M.

Reim, M.; Lax, F.; Lichte, H. and Turss, R. (Marburg/Lahn):

Steady State Levels of Glucose in the Different Layers of the Cornea,

Aqueous Humor, Blood and Tears in vivo 39

Rentsch, R.: vide Witmer, R.

Rintelen, F. (Basel):

Über Erblindungsursachen in der Schweiz 223

Schumacher, H. (Mainz):

Plasma-Elektrophorese bei Patienten mit Glaukom und intraokularen

Entzündungen 51

Snell, C. A. R. D.: vide Brienen, J. A. Sobanska, Maria (Lodz):

Linsenkapselpunktion als Therapiemethode der kongenitalen Linsen-

subluxation bei Kindern und Jugendlichen 564

Speiser, P.: vide Martenet, A. C.

Spinelli, D.; Molfetta, V. de et Maselli, E. (Milan):

La réponse électrorétinographique dans le décollement rétinien.

Comportement de Гonde c 387

Spinelli, D.: vide Molfetta, A. C.

Spinelli, D.: vide Miglior, M.

Streiff, E. B. (Lausanne):

Hommage au Professeur A. Franceschetti 163

Stucchi, C. A.; Rabinovicz, Th. et Faggioni, R. (Lausanne):

L'histopathologie de la dégénérescence en bandelette primaire et

familiale de la cornée 257

Turss, R.: $\quad$ vide Reim, M.

Vass, Z.: $\quad$ vide Kahán, I. L.

Verbeke, J.: vide Francois, J. 
Verluyten, P.: vide Francois, J.

Verrey, F. (Zurich): Cytologie de Thumeur aqueuse et invasion epithéliale de la chambre antérieure $\quad 310$

Vicari, S. (Lugano); Babel, J. (Geneve) et Cardinet, J. (Neuchâtel):

L'action du trihydroxyéthylrutoside dans la rétinopathie diabétique 21 Victoria-Troncoso, V.:

vide Francois, J. Witmer, R.; Iwamoto, T.; Rentsch, R. und Martenet, A. C. (Zurich):

Experimentelle Uveitis $\quad 301$

Witmer, R.: vide Maktenet, A. C.

Wüthrich, H. (Zollikofen):

Die Erziehung des blinden Kindes

239

Yobbágyi, P.: vide Francois, J.

Zuccoli, A. (Lugano):

Séméiologie ophtalmoscopique et techniques opératoires de quelques

cas de décollement de la rétine opérés en 1965

378

Proceedings - Verhandlungsberichte - Congrès

Schweizerische Ophthalmologische Gesellschaft, 59. General·ver-sammlung vom 15. bis 17.

September 1966 in Genf - Société Suissed'Ophtalmologie, 59e Assemblée générale du 15 au 17

Septembre 1966à Geneve 161

Further Studies and Other Information - Fortbildung und Orientierung -Perfectionnement et

Orientation

Amalric, P. (Albi, Tarn):

Nouvelles considerations concernant revolution et le traitement de

la rétinopathie diabétique 151

Bruckner, R. (Basel):

Augenmuskel-, Sehnerv- und Pupillenaffektionen beim Diabetes . 586

Jost, P.: $\quad$ vide Lang, J.

Lang, J.; Speiser, P.; Witmer, R. und Jost, P. (Zurich):

Geheilte Schielamblyopie: Normales Binokularsehen oder Al-ter-nieren?

Speiser, P.: vide Lang, J.

Witmer, R.: vide Lang, J.

Book. Reviews - Buchbesprechungen - Livres nouveaux 439

Index Rerum ad Vol. $154 \quad 592$

Index Autorum ad Vol. 154599

Alle Recnte, insbesondere das der Übersetzung in fremde Sprachen, vorbehalten.

Ohne ausdrückliche Genehmigung des Veriages ist es auch nicht gestattet, dieses Buch oder

Teile daraus auf photomechanischem Wege (Photokopie, Mikrokopie) zu vervielfältigen.

(C)

C. Copyright 1967 by S. Karger AG.

Basel. Printed in Switzerland by Buchdruckerei Friedrich Reinhardt AG.

Basel Cliches: Aberegg-Steiner \& Cie. AG.

Bern

und Steiner \& Cie. AG.. Basel 